\title{
Como o professor universitário aprende a ensinar? Um estudo na perspectiva da socialização profissional
}

How do university professors learn to teach? A study from the perspective of professional socialization

Este estudo insere-se no contexto das investigações que procuram utilizar conceitos sociológicos mais amplos como subsídio à compreensão de questões do campo da formação de professores. Nosso objetivo foi compreender como o professor universitário aprende a ensinar, os saberes e estratégias que constrói, tendo por categoria epistemológica interpretativa a socialização profissional. No desenvolvimento do estudo apoiamo-nos nas narrativas de oito professores da UFV/MG, sobre a história de vida profissional. A entrevista foi o instrumento utilizado na interlocução com os sujeitos. As análises foram articuladas em torno de três eixos - a formação, atuação e socialização profissional por meio dos quais exploramos conhecimentos e aprendizagens sobre o ensinar. Ao estudar "como o professor aprende a ensinar" vimos que os processos de aprendizagem da docência e de socialização profissional são ainda pouco explorados quando se considera o ensino superior. Em termos de políticas públicas e da instituição de atuação, o desenvolvimento profissional do professor aparece como espaços de autonomia. $\mathrm{O}$ professor universitário aprende a ensinar, na maioria das vezes, na prática, utilizando os referenciais aprendidos na formação específica $e$ na pós-graduação. A opção pelo magistério, no caso de alguns participantes da pesquisa, ocorreu porque a formação do pesquisador, no Brasil, acaba por direcionar o profissional para a universidade, onde ele irá se dedicar à pesquisa $e$, também, ao ensino e à extensão, se a Universidade se orientar pelo "modelo único" de instituição universitária. Entretanto, isso não nega a afinidade de muitos desses profissionais com a área de ensino. O professor do ensino superior, quando inicia a docência, também, sofre o impacto da relação com a cultura institucional. Em sua trajetória de atuação profissional, vai aprendendo a ensinar reproduzindo estratégias e práticas de seus antigos professores, mas busca, também, dar sua identidade à prática; na maioria das vezes, aprende o funcionamento da instituição solitariamente. A identidade profissional do professor universitário vai-se formando, inicialmente, por um ato de atribuição e de reconhecimento de seu papel, pela comunidade universitária e local e, posteriormente, por um ato de pertença, quando o professor já compreende a instituição, suas normas de funcionamento e se encontra socializado na profissão e na instituição. Por mais que a universidade esteja em processo contínuo de sucateamento, os professores investem e acreditam nela; acham que este ainda é um lugar de se trabalhar; que se tem reconhecimento, por parte de alunos, dos pares, e mesmo da comunidade local. O que se aponta, neste estudo: a necessidade de investimento no ensinar, por parte do professor universitário, tanto em termos de pesquisa e produções, quanto na criação de espaços em que o pesquisador-professor, se valendo da relativa autonomia que possui, possa estabelecer uma interlocução, mais cotidiana, com seus pares, sobre seus saberes, estratégias e embates na prática de ensino. Possivelmente, as tecnologias de informação e comunicação possam representar um dos recursos a ser utilizado no diálogo sobre o ensinar, sem que isto possa representar mais uma atribuição burocrática $e$ corrobore a intensificação do trabalho docente.

Alvanize Valente Fernandes Ferenc Tese (Doutorado), 2005

Programa de Pós-graduação em Educação

Universidade Federal de São Carlos, São Carlos <avalente@ufv.br>

PALAVRAS-CHAVE: educação superior. socialização. ensino. aprendizagem.

KEY WORDS: higher education. socialization. teaching. learning. PALABRAS CLAVE: educación superior. socialización. enseñanza. aprendizaje.

Recebido em 12/10/05. Aprovado em: 27/10/05. 


\section{As competências no cuidado com o diabetes melittus: contribuições à educação e comunicação em saúde}

Competencies in caring for patients with diabetes mellitus: contributions to healthcare education and communication

Um fosso separa o desenvolvimento teóricometodológico no campo da Educação $e$ Comunicação de sua prática nos serviços de saúde. Nestas instituições é, ainda, predominante o caráter prescritivo e centrado na transmissão de informações visando à mudança de hábitos, tomando-se como direta e quase mecânica a relação entre conhecimento e comportamento. É, também, preocupante que parcela expressiva das pesquisas no campo da Educação em Saúde voltadas para o controle do diabetes estejam orientadas por esta mesma lógica. Nesta pesquisa de natureza qualitativa, procuramos explorar as possibilidades teórico-

metodológicas de utilização do conceito de competências, como a capacidade de mobilizar saberes para dominar situações concretas. Nesta perspectiva, buscamos deslocar o tradicional binômio informação comportamento para uma articulação de outra natureza e mais próxima da relação: problema - saber - ação. Para isto, estudamos as competências para o autocuidado $e$ autocontrole no diabetes sob dois pontos de vista: aquelas requeridas, na opinião de diabetólogos, e aquelas efetivas, identificadas numa comunidade de diabéticos. As primeiras foram determinadas mediante levantamento documental técnico-científico e entrevistas com especialistas $e$ as segundas por meio de grupos focais e entrevistas em profundidade com diabéticos. Ao mesmo tempo, levantamos as dificuldades que os pacientes enfrentam para se cuidar, segundo as narrativas dos próprios diabéticos e os depoimentos dos especialistas. Reconhecemos 11 obstáculos ao autocuidado nos depoimentos destes últimos distribuídos em cinco subconjuntos, segundo sua relação com: a própria doença ou comorbidades, os próprios pacientes, a rede de apoio e a assistência prestada aos diabéticos.
As dificuldades do viver com diabetes, extraída dos discursos dos portadores, revelaram 26 campos problemáticos, que agrupamos em cinco categorias: o adoecer; o cotidiano e o cuidado-de-si; o cotidiano, o cuidado-de-si e o outro; o cotidiano, o cuidado-de-si e a rede de apoio; e, o cotidiano, o cuidado-de-si e os profissionais e os serviços de saúde. As competências requeridas conformaram um rol com 47 competências que envolvem cinco conjuntos de saberes e saberes-fazer, enquanto as competências efetivas compõem um quadro com 98 distintos saberes: saber, saber-fazer, saber-ser e saber-comunicar. Estes resultados mostram a expressiva riqueza de saberes mobilizados pelos diabéticos em sua experiência cotidiana de lidar com a doença, o que pode abrir possibilidades novas de diálogo com os profissionais de saúde, bem como aponta a relevância de reconhecer, disponibilizar e partilhar este "saber da experiência", no emergente campo da inteligência coletiva. A ampliação de vozes alcançada nesta investigação reforça a importância que um enfoque comunicacional pode fornecer para apoiar novos desenvolvimentos no campo da educação em saúde.

\section{Antonio de Pádua Pithon Cyrino} Tese (Doutorado), 2005 Faculdade de Medicina, Universidade de São Paulo. $<$ acyrino@gmail.com>

PALAVRAS-CHAVE: diabetes mellitus. autocuidado. educação em saúde. atitude do pessoal da saúde. educação do paciente.

KEY WORDS: diabetes mellitus. self care. health education. attitude of health personnel. patient education.

PALABRAS CLAVE: diabetes mellitus. educación en salud. actitud del personal de salud.educación del paciente. 


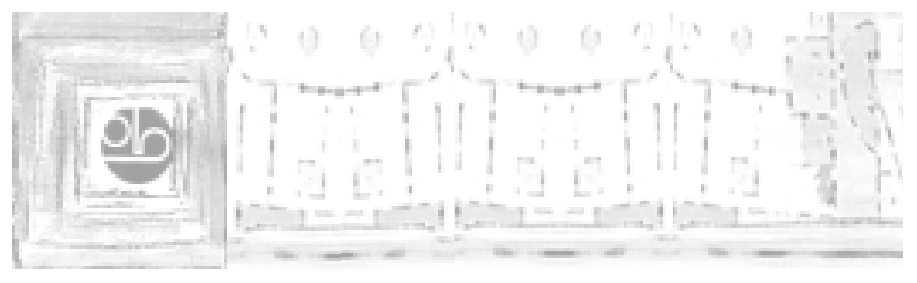

\section{Comunicação, promoção da saúde e democracia: políticas e estratégias de comunicação no Sistema Único de Saúde no Brasil}

Communication, the promotion of healthcare and democracy: the communication policies and strategies of Brazil's Single Healthcare System

Ressalta-se a relevância que as Comunicações assumem nas principais Conferências e encontros internacionais sobre Saúde dos Povos, quando o tema é tratado ao longo do tempo em sua dimensão instrumental.

Primeiramente, focaliza-se o "objeto" da chamada comunicação "em saúde". Para tanto, percorrem-se alguns dos enfoques teóricos em comunicação e em planejamento que em seu entendimento dão sustentação aos discursos e práticas institucionais no campo da Saúde Coletiva no Brasil. Busca, partindo do Postulado de coerência de Mário Testa, traçar relações entre as teorias da comunicação analisadas e os enfoques Normativo e Estratégico Situacional do Planejamento em Saúde, ressaltando a íntima relação da chamada "comunicação social" como o próprio "objeto-relação" do planejamento em Saúde como proposto por Marilene Castilho Sá em 1993. Descreve-se o estado da arte do que seriam no interior do postulado de coerência de Mário Testa, os princípios, políticas e estratégias de comunicação no contexto pós $8^{\text {a }}$ Conferência Nacional de Saúde e sua expressão no espaço do executivo - da Assessoria de Comunicação do Ministé- rio da Saúde. Como estratégias metodológicas, acompanham-se e analisam-se os processos de construção discursiva e de efetivação de algumas estratégias de comunicação entre governo e sociedade no campo da saúde coletiva, assumindo aí uma dada "posição de enunciação" $e$ de atuação política e se inscrevendo como sujeito da própria luta política. Como hipóteses de trabalho ressaltam-se as diferentes modalidades de organização dos processos de trabalho em comunicação no setor público (de saúde) e sua coerência com diferentes "estilos de governar" ou gerir a coisa pública; $e$ a necessidade de mudança de enfoque quanto às bases de sustentação teórica no campo das Teorias da comunicação com vistas a modalidades mais democráticas de gestão e novos "protocolos" de comunicação entre governos e cidadãos tradicionalmente entendidos, de forma reducionista, como emprego de técnicas de jornalismo, publicidade/propaganda, relações públicas e linguagens educativas - justapostos aos programas, decisões e imagens de um governo eleito - inclusive como estratégias de permanente sustentação $e$ fortalecimento de sua legitimidade. Utilizando entrevistas, coleta 
de dados sobre atividades e processos de trabalho de diferentes núcleos institucionais, bem como acompanhando reuniões do Conselho Nacional de Saúde e de uma efêmera Comissão Assessora para o campo da Comunicação, associa-se ao trabalho uma análise documental que se inicia em 1989. Há portanto, na construção, "uma espécie de conhecimento tácito compartilhado", que foi possibilitando as relações entre as Políticas de Comunicação governamentais e diferentes estilos de lidar com a gestão pública e que se manifestam a partir de uma rede de micro-decisões, movimentos, inventividades, astúcias: de movimentos quase imponderáveis com os quais a autora pretendeu dialogar.

A partir dos dados reunidos no trabalho de campo, são feitas relações preliminares entre
Modelos de Comunicação e Modelos de Democracia, propondo o que designou de "Padrões de Conflitualidade" na esfera pública para o ponto de partida de estudos transdisciplinares sobre o tema.

Áurea Maria da Rocha Pitta Tese (Doutorado), 2001 Escola de Comunicação, Universidade Federal do Rio de Janeiro <aureapitta@cict.fiocruz.br>

PALAVRAS-CHAVE: comunicação. promoção da saúde. SUS. KEYWORDS: communication. health promotion. SUS.

PALABRAS CLAVE: comunicación. promoción de la salud. SUS.

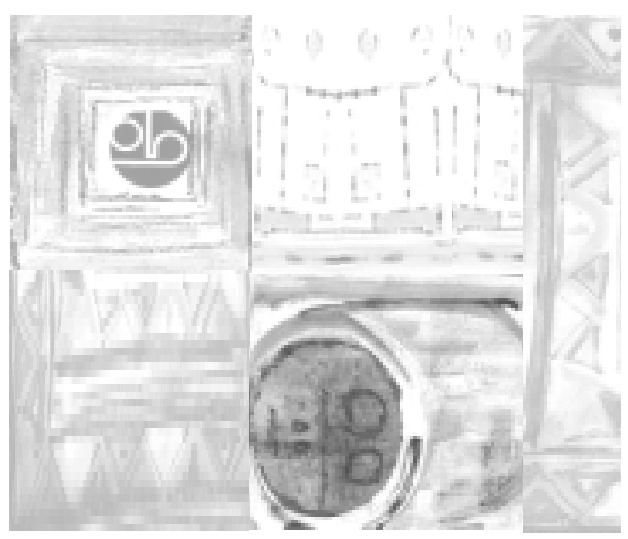




\section{Epistemologia contemporânea e saúde: a luta pela verdade e as práticas terapêuticas}

Contemporary epistemology and healthcare: the struggle for truth and therapeutic practices

Vários dilemas relacionais, éticos e terapêuticos da atenção à saúde biomédica estão relacionados com aspectos da epistemologia hegemônica aplicada na saúde, baseada num ideário positivista, cartesiano, representacionista $e$ mecanicista, proveniente da física clássica. Esse ideário também dificulta o relacionamento da biomedicina com os curadores não-científicos. Todavia, autores contemporâneos têm introduzido mudanças na epistemologia das ciências naturais, que, por hipótese, podem contribuir para amenizar ou solucionar alguns desses dilemas.

O trabalho consistiu do estudo, discussão e apresentação de sete autores representativos dessas mudanças, a saber, Popper, Lakatos, Kuhn, Fleck, Feyerabend, Maturana e Latour; uma reflexão sobre os possíveis desdobramentos dessas novas idéias epistemológicas na área da saúde (ensino e pesquisa, clínica e saúde coletiva); o delineamento de tendências extraídas desses autores e o esboço de um enfoque epistemológico neles baseado.

Como resultado, três tendências epistemológicas despontaram: a superação do positivismo mecanicista na saúde, a construção de uma nova visão epistemológica dita "coconstrutivista” - em que a realidade é vista como co-construída pelos sujeitos em interação com o mundo - e a reposição do homem, seus coletivos $e$ sua vida no centro da cena epistemológica. Isso, aplicado à saúde, mostra-se facilitador de mudanças na relação dos terapeutas com o saber biomédico e seus limites, $e$ indutor de melhorias no relacionamento da biomedicina com os pacientes e com os curadores não-científicos.

Ao final, derivando do enfoque coconstrutivista, é proposta uma combinação da noção de "tradição", de Feyerabend, com as idéias de estilo e coletivo de pensamento de Fleck e com visão não-moderna de Bruno Latour, para o reconhecimento, estudo e aplicação de medicinas ou racionalidades médicas distintas de forma simétrica, sem adesão a priori a uma delas. A partir dessa combinação é possível reconhecer estruturas sócio-cognitivas especializadas de saber/prática em saúde-doença ("tradições de cura") portadoras, por hipótese, de eficácia, passíveis de estudo, comparação e oferta como recurso terapêutico à população. Nessa proposta, a biomedicina é uma dessas tradições de cura.

Charles Dalcanale Tesser Tese (Doutorado), 2004

Departamento de Medicina Preventiva e Social, Faculdade de Ciências Médicas, Universidade Estadual de Campinas. <charlestesser@terra.com.br >

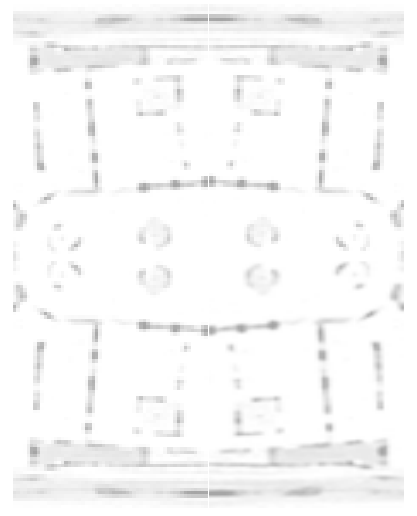

PALAVRAS-CHAVE: medicina. prática. saúde pública. medicina alternativa. epistemologia.

KEY WORDS: medicine. pratice. public health. alternative medicine. epistemology.

PALABRAS CLAVE: medicina. práctica. salud publica. medicina alternativa. epistemología.

Recebido em 06/10/05. Aprovado em: 18/10/05. 


\section{Estudos em Odontologia no SUS: educação do profissional de saúde bucal}

Studies in Dentistry in SUS: education of the professional of oral health

O objetivo deste trabalho foi discutir a educação do cirurgião-dentista para a atuação no Sistema único de Saúde (SUS). Para tanto, partiu-se de uma análise documental e teórica de contextualização histórica e conceituação da Odontologia enquanto prática de ensino, profissão e inserção no SUS.

Tendo como base essa análise, realizou-se uma pesquisa descritiva, na qual foram utilizados questionários e entrevistas como procedimentos de coleta de dados. Por meio desses procedimentos buscou-se identificar junto ao profissional graduado em Odontologia pela PUC-Campinas, que exerce função em setor público, sua percepção sobre a educação para o trabalho odontológico exercido no sus.

A partir da análise dos dados coletados verificou-se a necessidade de alguns elementos na educação do profissional de saúde bucal para atuação no SUS, os quais foram categorizados da seguinte maneira: 1. a diversificação dos cenários de ensino/ aprendizagem, a fim de favorecer a articulação entre a teoria e a prática e a priorização de situações reais de aprendizagem; 2. a construção de sujeitos com visão crítica $e$ capazes de articular ação e proposição; 3 . a ênfase na integralidade da atenção à saúde, no exercício multiprofissional e na compreensão/ aplicação dos conhecimentos técnico-científicometodológicos próprios da saúde coletiva. Portanto, o estudo revela a complexidade do desafio imposto às Universidades pela mudança na educação de cirurgiões-dentistas. Transformar o processo de educação desses profissionais significa mudar as concepções de saúde e educação e suas práticas, as relações entre cirurgiões-dentistas e população, entre cirurgiões-dentistas e demais profissionais da saúde, entre docentes e estudantes.

Gustavo Nicolini Fernandes Dissertação (Mestrado), 2004 Faculdade de Educação, Pontifícia Universidade Católica de Campinas <fernandesgustavo@yahoo.com.br>

PALAVRAS-CHAVE: cirurgiões-dentistas. saúde bucal. SUS. educação superior.

KEY-WORDS: dentists. oral health. SUS. higher education. PALABRAS CLAVE: dentistas. salud bucal. SUS. educación superior. 


\section{Representações de mulheres idosas usuárias de um serviço de saúde: relações entre consumo de calmantes, gênero e envelhecimento}

Representations of elderly women and users of a healthcare service: the relationship between consumption of tranquillizers, gender and aging

Os calmantes benzodiazepínicos são intensamente consumidos no mundo e no Brasil, principalmente por mulheres com idade igual ou superior a sessenta anos. O consumo de calmantes envolve fatores sociais, econômicos e culturais, fazendo com que a maneira de utilização seja adaptada à realidade de determinados grupos sociais.

Visando analisar as representações de mulheres idosas sobre o tema, fizemos uso, mediante abordagem qualitativa, de entrevistas semi-estruturadas com dezoito mulheres idosas, pertencentes a classes populares e consumidoras desses medicamentos há mais de um ano. Observamos que o modo de consumir calmantes é construído socialmente e essa construção é formada mediante o tempo de uso, da aproximação com os serviços de saúde, da classe social à qual pertencem as consumidoras, da definição social do papel da mulher na sociedade (no sentido de gênero) e do envelhecimento de seus usuários, acompanhado de experiências e conhecimentos sobre os medicamentos.

As mulheres entrevistadas mostraram ter autonomia e conhecimento sobre o uso dos calmantes, sentindo-se capazes de utilizar, indicar, emprestar, ou não, esses medicamentos, de acordo com suas concepções.

Reginaldo Teixeira Mendonça Dissertação (Mestrado), 2005. Universidade de São Paulo - USP Faculdade de Medicina de Ribeirão Preto Departamento de Medicina Social Programa de Pós-Graduação em Saúde na Comunidade <reginaldo_mendonca@ig.com.br>

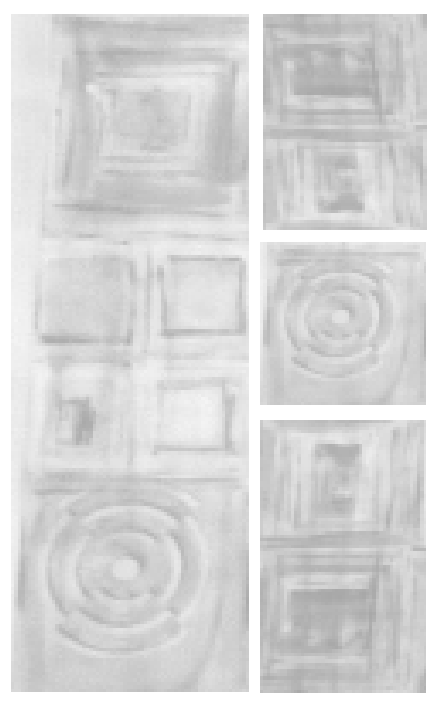

PALAVRAS-CHAVE: tranquilizantes. envelhecimento. gênero. medicina popular.

KEY WORDS: tranquilizing agents. aging. gender. folk medicine.

PALABRAS CLAVE: tranquilizantes. envejecimiento. gênero. medicina popular. 